Documentation et bibliothèques

DOCUMENTATION BIBLIOTHËQUES

\title{
Centre de documentation sur les bibliothèques, Bibliothèque nationale du Canada
}

\section{Marion C. Wilson}

Volume 19, numéro 1, mars 1973

URI : https://id.erudit.org/iderudit/1050959ar

DOI : https://doi.org/10.7202/1050959ar

Aller au sommaire du numéro

Éditeur(s)

Association pour l'avancement des sciences et des techniques de la documentation (ASTED)

ISSN

0315-2340 (imprimé)

2291-8949 (numérique)

Découvrir la revue

Citer cet article

Wilson, M. C. (1973). Centre de documentation sur les bibliothèques, Bibliothèque nationale du Canada. Documentation et bibliothèques, 19(1), 20-22. https://doi.org/10.7202/1050959ar
Résumé de l'article

Description du Centre et de ses collections faites surtout de rapports, d'articles de périodiques, de bulletins de nouvelles, de textes de lois, de renseignements sur des projets de recherche, etc..; énumération des services (référence, orientation) et des publications (Nouvelles acquisitions, Réunions à venir), tout en mettant l'accent sur le rôle national et international du centre.
Tous droits réservés (C) Association pour l'avancement des sciences et des techniques de la documentation (ASTED), 1973
Ce document est protégé par la loi sur le droit d'auteur. L'utilisation des services d'Érudit (y compris la reproduction) est assujettie à sa politique d'utilisation que vous pouvez consulter en ligne.

https://apropos.erudit.org/fr/usagers/politique-dutilisation/ 


\section{Centre de documentation sur les bibliothèques, Bibliothèque nationale du Canada}

\author{
Marion C. Wilson \\ Bibliothèque nationale \\ Ottawa
}

Description du Centre et de ses collections faites surtout de rapports, d'articles de périodiques, de bulletins de nouvelles, de textes de lois, de renseignements sur des projets de recherche, etc..; énumération des services (référence, orientation) et des publications (Nouvelles acquisitions, Réunions à venir), tout en mettant l'accent sur le rôle national et international du centre.

La direction de la recherche et de la planification a été créée en 1970 "afin d'effectuer la recherche opérationnelle recommandée dans le Rapport sur l'étude de l'automatisation des services et de coordonner la planification visant à uniformiser le réseau de communications avec les autres bibliothèques, canadiennes et étrangères". " Au moment où la nouvelle direction en était au stade de l'organisation, on a reconnu l'impérieux besoin de mettre à la disposition du personnel de la Bibliothèque nationale les renseignements les plus récents possibles sur la recherche et le développement dans le domaine de la bibliothéconomie, particulièrement en ce qui concerne l'automatisation des travaux de bibliothèque. On a également reconnu la nécessité, à l'échelle nationale, d'un centre d'information et de documentation dans le vaste domaine qu'englobent les termes "bibliothéconomie et sciences de l'information". En conséquence, on a décidé d'établir le Centre de documentation sur les bibliothèques à l'intérieur de la nouvelle direction, dès sa formation en novembre 1970.

\section{Les collections}

Dès le début, les fonctions du centre ont été conçues en termes très généraux et on s'est mis à collectionner de la documentation et de l'information, déjà parues ou inédites, sur un large éventail de sujets. Même si, en raison des projets d'automatisation de la $\mathrm{Bi}$ bliothèque nationale et de la nomination, en 1971 et en 1972, de trois groupes de travail, l'un sur la normalisation du catalogage, un autre sur une version canadienne du MARC et un dernier sur le Catalogue collectif cana-

1. Rapport du directeur général de la Bibliothèque nationale, 1971, Ottawa, Information Canada, 1971. dien, on a dû accorder beaucoup d'importance à la recherche de documentation apte à répondre aux besoins d'information du personnel de la Bibliothèque nationale et des membres des groupes de travail, on n'a pas pour autant négligé la nécessité d'une source centrale d'information sur les bibliothèques canadiennes et les événements les concernant.

Les sources existantes de documentation sur l'activité des bibliothèques et les recherches en bibliothéconomie sont nombreuses et variées, allant des ouvrages de référence ordinaires aux communications orales en provenance de sources bien informées, à l'intérieur ou à l'extérieur du monde des bibliothèques. Des études et des rapports sur des travaux de recherche, des rapports sur l'état d'avancement des recherches, des études effectuées par des bibliothèques particulières avant tout pour leurs propres services, des articles parus dans des périodiques, des documents du gouvernement, des coupures de journaux, des bulletins de nouvelles émis par des bibliothèques ou des associations des secteurs de la bibliothéconomie et de l'informatique, des travaux de réunions ou de conférences, des documents préparés à l'intention de groupes de travail ou en vue de colloques, des discours et des communiqués, voilà autant de sources utiles d'informations à jour, et le centre s'efforce de les exploiter toutes. Depuis 1970, le centre reçoit une sélection variée des rapports du Educational Resources Information Center/Clearinghouse on Library and Information Sciences (ERIC/CLIS), dont la plus grande partie sur microfiches. Ces microfiches peuvent être utilisées sur place ou empruntées par prêts entre bibliothèques. L'an dernier, on a désigné le Centre de documentation sur les bibliothèques pour recevoir tous les documents relatifs aux conférences de la Fédération internationale des associations de bibliothécaires (FIAB). Le centre vient de recevoir le texte des communications présentées à la conférence de Budapest en 1972. ${ }^{2}$

On collectionne systématiquement certaines formes de documents, notamment les textes de lois et de règlements relatifs aux bibliothèques, les rapports des bibliothèques canadiennes de toute catégorie, les programmes des écoles de bibliothéconomie et des cours à l'intention des bibliotechniciens, les catalogues des fabricants et des fournisseurs de matériel de bibliothèques et d'ordinateurs. On commence à collectionner les plans des immeubles logeant des bibliothèques afin de compléter une collection remise à la Bibliothèque nationale par la Canadian Library Association.

La documentation sur les services automatisés de bibliothèques est particulièrement précieuse, et le centre s'est occupé en 1972

2. On peut obtenir la liste des communications en s'adres sant au Centre de documentation sur les bibliotheques. Le centre sera bientôt en mesure de fournir des photocopies de chacune des communications. 
de mettre à jour les renseignements sur les programmes destinés à des systèmes automatisés dans les bibliothèques des universités canadiennes, recueillis par l'Association canadienne des bibliothèques de collège et d'université après son colloque sur l'automatisation tenu en 1970, ce qui permettra d'établir un système d'échange de programmes. On recueille aussi de la documentation sur l'automatisation des systèmes dans d'autres bibliothèques et des formulaires sont disponibles au centre. Le centre est abonné à un service canadien de coupures, et il obtient de cette source une foule de renseignements utiles sur des bibliothèques particulières et de données biographiques sur les bibliothécaires et les informaticiens du Canada. La Division des acquisitions de la bibliothèque reçoit régulièrement des recommandations relatives à l'achat d'imprimés, y compris les abonnements à des périodiques, et, depuis la création du centre, la Bibliothèque nationale augmente à un rythme accéléré ses collections de monographies et de périodiques sur la bibliothéconomie et l'informatique.

\section{Recherches en matière de documentation et de bibliothéconomie}

Le centre s'occupe, depuis sa création, de recueillir des renseignements et des rapports sur des recherches concernant la bibliothéconomie et la documentation. Lors de sa conférence de juin 1970, la Canadian Library Association a adopté une résolution recommandant l'inclusion de cette activité dans les fonctions du centre et la publication d'une liste annuelle des travaux de recherche terminés ou en cours dans ce domaine, ce qui semble confirmer l'utilité possible de cette activité. Au début de 1972, le Ministère des affaires extérieures a reçu du Secrétariat de l'Unesco la demande suivante: la désignation au Canada d'une institution nationale chargée de "rassembler dans le pays et mettre à la disposition du Secrétariat de l'Unesco, éventuellement en ouvrant et en tenant un registre national de projets et comptes rendus de recherche et en faisant office de dépôt national pour les comptes rendus de recherche, (i) des données sur les projets et comptes rendus de recherche; (ii) des exemplaires des comptes rendus de recherche; les premières et les secondes étant des éléments nécessaires du Système international d'information sur les recherches en matière de documentation (ISORID)". En mars 1972, le Centre de documentation sur les bibliothèques a été désigné comme Centre national de transfert de l'information du Canada, dans le cadre du système ISORID.

Le centre a reçu des formules de rapport accompagnées d'un exposé des Principes directeurs à l'intention des Centres nationaux de transfert de l'information sur la création du Système international d'information sur les recherches en matière de documentation (ISORID). On distribuera le plus tôt possible des exemplaires des Principes directeurs et des formules aux éventuels participants canadiens à ISORID. Tout lecteur intéressé peut aussi se procurer ces documents immédiatement en en faisant la demande au Centre de documentation sur les bibliothèques. Voici comment on a résumé l'objectif de I'ISORID: "Un système d'information établi par l'Unesco, en coopération avec la Fédération internationale de documentation ( $F I D)$ et chargé de rassembler, d'organiser, d'analyser et de diffuser des informations sur les activités et les efforts de recherches dans les domaines de la documentation, des bibliothèques et des archives." On ajoute que l'expression "documentation" désigne toutes sortes d'activités que comporte le processus d'ensemble du rassemblement, de l'organisation, de l'analyse et de la dissémination de l'information, c'est-à-dire qu'elle comprend également l'informatique, la bibliothéconomie et les domaines connexes, par exemple l'analyse et la création de systèmes d'information, les banques de données, la linguistique dans le domaine de la documentation, la traduction mécanique, les moyens et les matériaux, la mise au point ei l'application spéciale d'un nouvel outillage technique et la terminologie de la documentation. En plus des thèses et des recherches pures et appliquées, au niveau du doctorat, I'ISORID s'occupe de "certaines autres activités intéressant la recherche et le développement (sur lesquelles ne portent pas en général les autres services de résumés analytiques et d'indexation) telles que: les études sur l'état d'avancement des recherches (state-of-the-art surveys); les études de rentabilité; la démonstration, l'organisation et la planification, la comparaison des systèmes ou méthodes d'information; les études portant sur les utilisateurs; les efforts d'innovation; les nouvelles méthodes de formation, etc."

\section{Les services et les publications}

L'objectif général du centre est d'offrir un service de rassemblement et de diffusion de données portant sur la recherche et le développement en bibliothéconomie et dans les domaines connexes. Le centre met l'accent sur les projets conçus au Canada, sur les bibliothèques canadiennes et leurs activités. Des services de référence et de documentation ainsi qu'un service d'orientation sont mis à la disposition du personnel de la Bibliothèque nationale et de celui des bibliothèques des organismes gouvernementaux, des universités, des services de recherche, et des bibliothèques publiques, régionales et spécialisées, afin de favoriser un échange efficace de renseignements entre les bibliothèques canadiennes et de constituer une source centrale d'information pour l'étranger. Les institu- 
tions et les particuliers peuvent se prévaloir de ces services. Un service officieux d'information courante est mis à la disposition du personnel de la Bibliothèque nationale. C'est ainsi que, par exemple, le personnel du centre fera parvenir au Coordonnateur des catalogues collectifs une photocopie de tout article, de tout rapport de recherche relatifs aux catalogues collectifs. Ce service, qui tente d'aller au devant des demandes, est axé sur les activités et les projets des divers services, divisions et sections et sur les besoins des membres du personnel. Même si ses services doivent avant tout répondre aux besoins de la Bibliothèque nationale, à mesure qu'il est devenu mieux connu, le centre a reçu une demande croissante d'information de l'extérieur. Des ministères et des organismes du gouvernement, des bibliothécaires et des chercheurs particuliers, des associations et des institutions nationales et internationales ont présenté des demandes de renseignements et de documentation.

Le centre prépare une liste intitulée Nouvelles acquisitions dans laquelle sont répertoriés par sujets les documents ajoutés aux dossiers, les ouvrages de référence conservés au centre et les monographies dans le domaine de la bibliothéconomie et des sciences de l'information ajoutées à la collection générale de la bibliothèque. On prépare de temps à autre des bibliographies sélectives annotées sur des sujets susceptibles d'intéresser les membres du personnel. Cette liste et une liste intitulée Réunions à venir sont distribuées chaque mois au personnel professionnel.

Le centre doit entreprendre la compilation d'un répertoire des bibliothèques du gouvernement fédéral basé sur les données recueillies dans le cadre d'une enquête détaillée présentement en cours. ${ }^{3}$ Le répertoire devrait paraître en 1974. II sera le premier d'une série de volumes consacrés aux divers genres de bibliothèques canadiennes. Le centre pourrait aussi être appelé à entreprendre un répertoire biographique des bibliothécaires et des spécialistes de l'information canadiens.

D'ici quelques mois, le centre devrait être en mesure de compiler une première liste de projets de recherche en cours dans le domaine de la bibliothéconomie et de la documentation. On n'a pas encore décidé du format, de la diffusion, ni de la périodicité de cette liste puisque l'on ne sait pas encore quel genre de renseignements le centre recevra en temps que Centre national de transfert de l'information dans le cadre du système ISORID. Les activités du centre dépendront de la coopération que lui accorderont chercheurs et bibliothécaires.

\section{La coopération entre les bibliothèques canadiennes}

On parcourt chaque mois quelque cinquante à soixante périodiques et bulletins de nouvelles et des centaines de coupures pour y repérer des indices sur la documentation disponible, et on présente par lettre ou par téléphone de nombreuses demandes de renseignements à des sources probables. Le centre est abonné au service de diffusion sélective d'informations (CAN/SDI) de la Bibliothèque scientifique nationale et au service similaire qu'offre la Bibliothèque nationale. Le personnel assiste à des conférences organisées pour les gens de la profession et un début modeste de visites aux bibliothèques et aux écoles de bibliothéconomie est en cours. Néanmoins, le centre compte beaucoup sur la collaboration de collègues et fait des demandes d'études et de rapports. Maintenant que le centre est un organisme national de transfert de l'information dans le cadre du projet ISORID du Secrétariat de I'Unesco, la collaboration entre les bibliothèques canadiennes revêt une importance plus grande encore. Les études et les rapports de nature confidentielle ou destinés au seul usage de telle ou telle bibliothèque peuvent porter la mention "diffusion restreinte" et ne seront pas mis à la disposition d'une autre institution ou personne sans une permission expresse dans ce sens.

Récipiendaire de la bourse Grolier:

Daniel Allaire de Sherbrooke Etudiant à l'université de Montréal

\section{ERRATUM}

Livraison de décembre 1972 du Bulletin p. $281,2^{\mathrm{e}}$ colonne, dernière ligne: lire: "de la qualité ..." et non: "de la quantité ..." 